\title{
Resistência antimicrobiana dos uropatógenos em pacientes ambulatoriais, 2000-2004
}

\author{
Antimicrobial resistance of uropathogens \\ among outpatients, 2000-2004
Camila Ribeiro Koch², Juliana Camurça Ribeiro², Otávio Haygert Schnor², Bruno Sudbrack Zimmermann², Fernanda Melo Müller ${ }^{2}$, Juliana D’ Agostin², Vívian Machado² e Linjie Zhang ${ }^{1}$

\begin{abstract}
RESUMO
Foi realizado um estudo retrospectivo, baseado no banco de dados eletrônico de um hospital universitário, com objetivo de investigar a prevalência dos germes causadores e suas suscetibilidades aos antibióticos em adultos (idade $>18$ anos), com infecção do trato urinário atendidos ambulatorialmente. Foram identificados 957 exames de urocultura positiva no período entre janeiro de 2000 e dezembro de 2004. Escherichia coli, Proteus mirabillis e Klebsiella $s p$ foram três principais bactérias causadoras. Sulfametoxazol-trimetropim apresentou a maior (46,9\%) prevalência de resistência bacteriana seguida por cefalotina (46,7\%), ácido nalidíxico (27,6\%) e nitrofurantoína (22,3\%). Durante o período estudado, 0 ácido nalidíxico apresentou um aumento anual de 5,9\% na taxa de resistência bacteriana ( $\mathrm{p}=0,02)$. Ciprofloxacina mostrou também a tendência de aumento, com um crescimento anual de 3,3\% ( $\mathrm{p}=0,07)$. Este estudo demonstrou que os antibióticos amplamente recomendados no tratamento empírico da infecção do trato urinário em adultos apresentaram altas taxas de resistência bacteriana na população estudada.
\end{abstract}

Palavras-chaves: Infecção do trato urinário. Resistência bacteriana. Antibióticos. Urocultura. Estudo retrospectivo.

\begin{abstract}
A retrospective study based on the electronic database of a university hospital was carried out to investigate the prevalence of etiological agents and their susceptibilities to antibiotics, among adult outpatients ( $>18$ years old) with urinary tract infections. Nine hundred and fifty-seven positive urine cultures were identified between January 2000 and December 2004. Escherichia coli, Proteus mirabilis and Klebsiella sp were the three principal bacterial etiological agents. Trimethoprim-sulfamethoxazole presented the highest prevalence of bacterial resistance (46.9\%), followed by cefalotin (46.7\%), nalidixic acid (27.6\%) and nitrofurantoin (22.3\%). Over the study period, nalidixic acid presented annual increases of $5.9 \%$ in the rate of bacterial resistance $(\mathrm{p}=0.02)$. Ciprofloxacin also showed an increasing trend, of $3.3 \%$ per year $(\mathrm{p}=0.07)$. This study demonstrated that the antibiotics that are widely recommended for empirical treatment of urinary tract infection in adults presented high rates of bacterial resistance among the population studied.
\end{abstract}

Key-words: Urinary tract infection. Bacterial resistance. Antibiotics. Urine culture. Retrospective study.

A infecção do trato urinário (ITU) é uma condição freqüiente no sexo feminino, visto que 20 a 48\% das mulheres apresentam pelo menos um episódio de ITU durante a vida ${ }^{714}$. 0 agente causador mais freqüente de ITU adquirida na comunidade, independente da faixa etária, é a Escherichia coli, cuja prevalência varia de 54 e $81 \%$. Outras bactérias mais prevalentes de ITU não complicada em adultos incluem Klebsiella spp, Proteus spp, Enterococus spp e Enterobacter spp $p^{3514}$.
A escolha empírica dos antibióticos no tratamento da ITU é determinada por alguns fatores como o germe causador mais provável, o padrão local da resistência bacteriana, a história prévia de uso de antibióticos pelo paciente, a imunidade do paciente, o custo, a disponibilidade e a farmacocinética do fármaco. No Brasil, os antibióticos recomendados para o tratamento empírico da ITU adquirida na comunidade em adultos são sulfametoxazol/ trimetroprima (SMZ-TMP), quinolonas (norfloxacina ou

\footnotetext{
1. Departamento Materno-Infantil, Fundação Universidade Federal do Rio Grande, Rio Grande, RS. 2. Acadêmicos do Curso de Medicina, Fundação Universidade Federal do Rio Grande, Rio Grande, RS.

Endereço para correspondência: Dr. Linjie Zhang. Dept ${ }^{\circ}$ Materno-Infantil/FURG. Visconde Paranaguá 102, Centro, $96200-190$ Rio Grande, RS.

Tel: $55533233-0311$

e-mail: zhanglinjie63@yahoo.com.br

Recebido para publicação em: 28/01/2008

Aceito em: 11/06/2008
} 
ciprofloxacina), cefalosporinas de $1^{\mathrm{a}}$ ou $2^{\mathrm{a}}$ gerações, amoxiciclina/ clavulanato ou nitrofurantoína ${ }^{76}$. No entanto, o crescimento da resistência antimicrobiana dos uropatógenos deixa dúvida sobre a validade dessas recomendações ${ }^{2}$.

Estudos realizados em diversas localizações do Brasil demonstram alta taxa de resistência de Escherichia coli a amoxacilina/ampicilina, sulfametoxazol-trimetoprim e cefalotina ${ }^{25}$. Porém, o padrão da resistência antimicrobiana dos uropatógenos também pode variar expressivamente com o local do estudo ${ }^{4}$. 0 conhecimento do padrão local da resistência bacteriana é de fundamental importância na orientação da escolha adequada dos antibióticos empíricos para tratamento dos pacientes com ITU. Além disso, no Brasil ainda são escassos os estudos que investigam a evolução temporal da resistência antimicrobiana dos uropatógenos em pacientes adultos com ITU adquirida na comunidade.

Temos como objetivos investigar os agentes mais prevalentes e suas suscetibilidade aos antibióticos em adultos com ITU, atendidos no nível ambulatorial de um hospital universitário no extremo sul do Estado do Rio Grande do Sul, durante um período de 5 anos.

\section{MATERIAL E MÉTODOS}

Este estudo retrospectivo foi realizado baseado no banco de dados eletrônico do Hospital Universitário da Fundação Universidade Federal do Rio Grande (FURG). 0 hospital universitário da FURG é um dos dois hospitais do município de Rio Grande, com aproximadamente 200.000 habitantes, localizado no extremo sul do Estado do Rio Grande do Sul. Foram incluídas no estudo as uroculturas com resultados positivos de adultos (idade > 18 anos) provenientes dos ambulatórios do hospital, no período compreendido entre janeiro de 2000 e dezembro de 2004.

Todos os exames foram realizados no Laboratório de Análises Clínicas do Hospital Universitário da FURG, baseado nas recomendações da National Committee for Clinical Laboratory Standards ${ }^{13}$. Utilizaram-se amostras de urina homogeneizadas e submetidas à coloração pelo método de Gram. A contagem de colônias foi feita usando o sistema Culturin ${ }^{\circledR}$ que contém dois meios de cultura: ágar cistina lactose deficiente em eletrólitos (CLED) e ágar MacConkey. Para amostras colhidas com micção espontânea foram consideradas negativas as uroculturas que apresentaram contagens menores que 100.000 unidades formadoras de colônia por mL (UFC/mL). Para 100.000 ou mais UFC/mL, foi identificada bactéria e realizado antibiograma. Para coletas através de punção suprapúbica qualquer contagem foi considerada crescimento bacteriano e procederam-se a identificação e o antibiograma, no qual a susceptibilidade aos antimicrobianos foi avaliada pelo método de difusão em disco com o uso de critérios de interpretação padrão $0^{11} 1213$.

0 processamento de dados e a análise estatística foram realizados utilizando o programa Stata 8.0 (Stata Corporation, Texas, USA, 2003). 0 teste de $\chi^{2}$ foi utilizado para comparação da prevalência da resistência bacteriana entre os grupos. 0 teste de regressão linear foi utilizado para verificar a tendência temporal da resistência bacteriana no período 2000-2004. Valor de $p<0,05$ foi considerado estatisticamente significativo para testes bi-caudais.

\section{RESULTADOS}

Foram identificados 957 exames de urocultura positiva, em $778(81,3 \%)$ mulheres e $179(\mathbf{1 8 , 7 \% )}$ homens. A idade dos pacientes variou de 19 a 96 anos, com média de 52,9 anos (IC 95\%: 51,79-54,07 anos).

Escherichia coli, Proteus mirabillis e Klebsiella sp foram três bactérias mais freqüentemente identificadas nas 957 amostras de urina, cujas prevalências foram 66,2\%, 8,4\% e 5,6\% respectivamente. A proporção do Proteus e da Klebsiella como agente etiológico da ITU foi maior em pacientes masculinos, comparados com as mulheres, mas não houve diferença significativa quanto à distribuição etiológica conforme a idade do paciente (Figuras 1A e B). Foram incluídas na análise final somente 762 uroculturas com presença de uma das três principais bactérias.

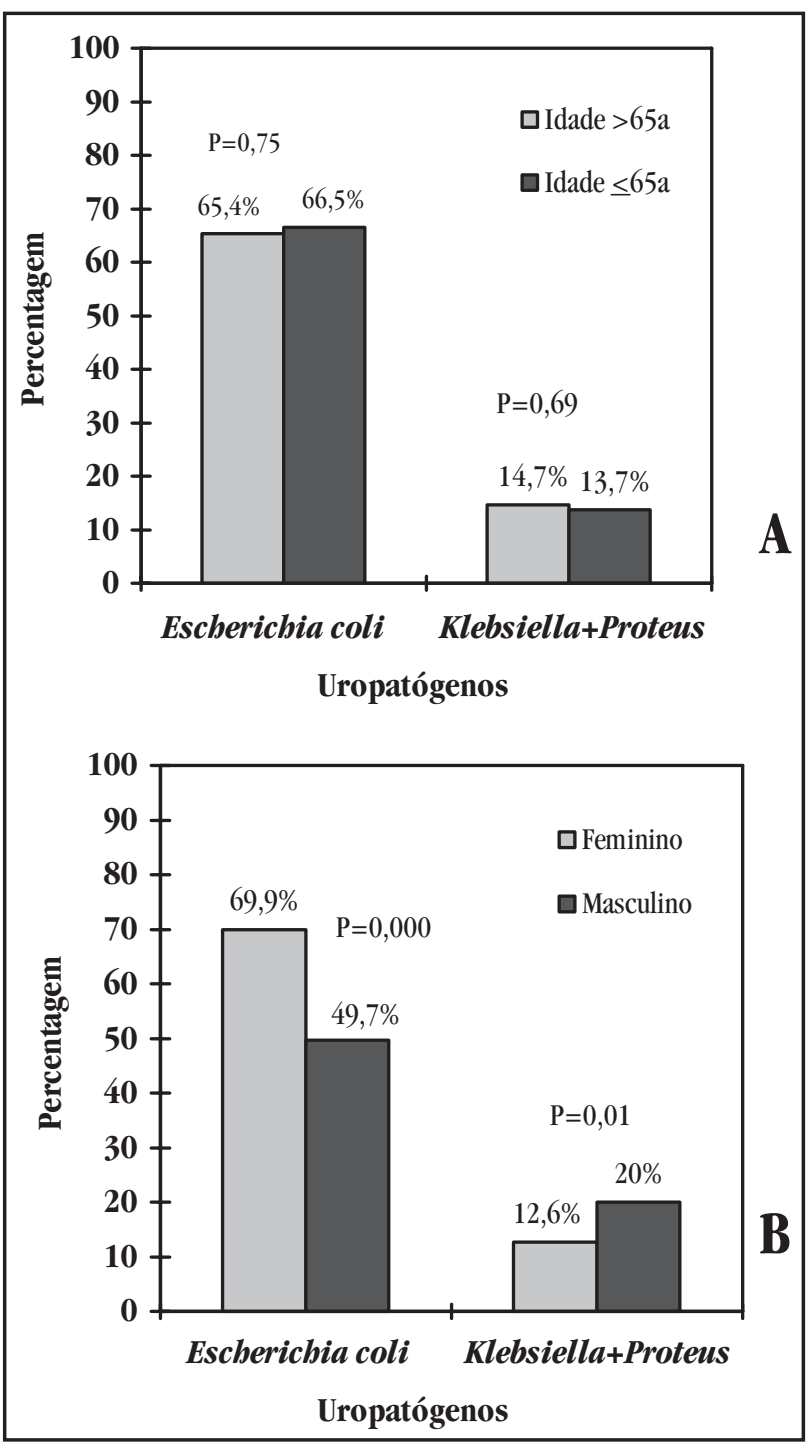

Figura 1 - A e B: Distribuição etiológica de infecção do trato urinário conforme idade e gênero do paciente. 
A Tabela 1 mostra as prevalências da resistência antimicrobiana das três bactérias uropatogênicas. A Klebsiella apresentou a maior prevalência da resistência bacteriana aos antibióticos, com uma média de 35\%, seguido de Proteus mirabilis $(33,1 \%)$ e Escherichia coli $(21,7 \%)$. 0 antibiótico que apresentou a maior prevalência da resistência bacteriana foi sulfametoxazol-trimetoprim como média $46,9 \%$, seguida por cefalotina (46,7\%), ácido nalidíxico $(27,6 \%)$ e nitrofurantoína $(22,3 \%) .0$ antibiótico que apresentou a menor prevalência da resistência bacteriana foi ceftriaxona como média 3,1\%, seguida por gentamicina $(12,2 \%)$, ciprofloxacina $(13,1 \%)$, e cefoxitina $(14,9 \%)$.

A Tabela 2 mostra a evolução temporal da resistência bacteriana aos antibióticos atestados durante o período estudado (2000-2004). Ácido nalidíxico foi o único antibiótico que apresentou aumento significativo de resistência bacteriana, com um crescimento anual de 5,9\% $(\mathrm{p}=0,02)$. Ciprofloxacina mostrou a tendência de aumento na taxa de resistência bacteriana, com um crescimento anual de 3,3\% $(\mathrm{p}=0,07)$. Os antibióticos que mantiveram alta taxa de resistência bacteriana durante o período estudado foram sulfametoxazol-trimetoprim, cefalotina e nitrofurantoína, em contraste com ceftriaxona que manteve baixa taxa de resistência bacteriana durante o mesmo período.

Observou-se que os pacientes com mais de 65 anos apresentaram maior taxa de resistência aos três antibióticos: ácido nalidíxico, nitrofurantoína e ciprofloxacina. Em relação ao gênero do paciente, os homens apresentaram maior taxa de resistência a todos os antibióticos atestados, com exceção da sulfametoxazol-trimetoprim (Tabela 3).

Tabela 1 - Resistência bacteriana aos antibióticos testados.

\begin{tabular}{|c|c|c|c|c|c|c|c|c|c|c|c|c|}
\hline \multirow[b]{2}{*}{ Antibiótico } & \multicolumn{3}{|c|}{ Escherichia coli } & \multicolumn{3}{|c|}{ Klebsiela } & \multicolumn{3}{|c|}{ Proteus } & \multicolumn{3}{|c|}{ Total } \\
\hline & $\mathrm{n}^{\circ}$ & $\mathrm{N}$ & $\%$ & $\mathrm{n}^{\circ}$ & $\mathrm{N}$ & $\%$ & $\mathrm{n}^{\circ}$ & $\mathrm{N}$ & $\%$ & $n^{\circ}$ & $\mathrm{N}$ & $\%$ \\
\hline Gentamicina & 75 & 617 & 12,2 & 8 & 52 & 15,4 & 7 & 79 & 8,7 & 90 & 748 & 12,0 \\
\hline Cefalotina & 273 & 612 & 44,6 & 30 & 53 & 56,6 & 44 & 78 & 56,4 & 347 & 743 & 46,7 \\
\hline Cefoxitina & 69 & 591 & 11,7 & 13 & 49 & 26,6 & 24 & 73 & 32,9 & 106 & 713 & 14,9 \\
\hline Nitrofurantoina & 88 & 613 & 14,4 & 30 & 52 & 57,7 & 48 & 80 & 60,0 & 166 & 745 & 22,3 \\
\hline Ácido nalidíxico & 156 & 620 & 25,2 & 24 & 53 & 45,3 & 28 & 80 & 35,0 & 208 & 753 & 27,6 \\
\hline Ceftriaxona & 13 & 616 & 2,1 & 3 & 51 & 5,9 & 7 & 79 & 8,9 & 23 & 746 & 3,1 \\
\hline Ciprofloxacin & 81 & 623 & 13,0 & 11 & 52 & 21,2 & 7 & 79 & 8,9 & 99 & 754 & 13,1 \\
\hline SMZ-TMP & 275 & 597 & 46,1 & 24 & 51 & 47,1 & 41 & 77 & 53,3 & 340 & 725 & 47,0 \\
\hline
\end{tabular}

SMZ-TMP: sulfametoxazol-trimetoprim, N: número total.

Tabela 2 - Evolução temporal da resistência bacteriana aos antibióticos em infecção do trato urinário.

\begin{tabular}{|c|c|c|c|c|c|c|c|c|c|c|c|c|c|c|c|c|}
\hline \multirow[b]{2}{*}{ Antibiótico } & \multicolumn{3}{|c|}{2000} & \multicolumn{3}{|c|}{2001} & \multicolumn{3}{|c|}{2002} & \multicolumn{3}{|c|}{2003} & \multicolumn{3}{|c|}{2004} & \multirow{2}{*}{$\begin{array}{l}\text { Mudança em } \\
\text { \%/ano (p valor) }\end{array}$} \\
\hline & $\mathrm{n}^{0}$ & $\mathrm{~N}$ & $\%$ & $\overline{n^{0}}$ & $\mathrm{~N}$ & $\%$ & $\mathrm{n}^{0}$ & $\mathrm{~N}$ & $\%$ & $\mathrm{n}^{0}$ & $\mathrm{~N}$ & $\%$ & $\mathrm{n}^{0}$ & $\mathrm{~N}$ & $\%$ & \\
\hline Gentamicina & 6 & 121 & 5,0 & 27 & 129 & 20,9 & 25 & 162 & 15,4 & 16 & 186 & 8,6 & 17 & 157 & 10,8 & $-0,03(0,98)$ \\
\hline Cefalotina & 67 & 126 & 53,2 & 76 & 126 & 60,3 & 58 & 157 & 36,9 & 83 & 184 & 45,1 & 67 & 157 & 43,7 & $-3,42 \quad(0,29)$ \\
\hline Cefoxitina & 11 & 127 & 8,7 & 15 & 87 & 17,2 & 26 & 165 & 15,8 & 27 & 183 & 14,8 & 30 & 158 & 19,0 & $+1,82(0,16)$ \\
\hline Nitrofurantoína & 32 & 124 & 25,8 & 35 & 129 & 27,1 & 30 & 164 & 18,3 & 37 & 180 & 20,6 & 34 & 155 & 21,9 & $-1,43(0,27)$ \\
\hline Ácido nalidíxico & 16 & 126 & 12,7 & 29 & 127 & 22,8 & 42 & 164 & 25,6 & 69 & 185 & 37,3 & 55 & 158 & 34,8 & $+5,87(0,02)$ \\
\hline Ceftriaxona & 2 & 119 & 1,7 & 3 & 130 & 2,3 & 7 & 165 & 4,2 & 6 & 182 & 3,3 & 6 & 157 & 3,8 & $+0,53(0,12)$ \\
\hline Ciprofloxacina & 4 & 127 & 3,2 & 13 & 130 & 10,0 & 26 & 165 & 15,8 & 35 & 186 & 18,8 & 23 & 153 & 15,0 & $+3,26(0,07)$ \\
\hline SMZ-TMP & 52 & 125 & 41,6 & 53 & 129 & 44,1 & 87 & 163 & 53,4 & 93 & 185 & 50,3 & 59 & 130 & 45,4 & $+1,67(0,40)$ \\
\hline
\end{tabular}

SMZ-TMP: sulfametoxazol-trimetoprim, N: número total.

Tabela 3 - Comparação de resistência antimicrobiana em relação à idade e gênero dos pacientes.

\begin{tabular}{|c|c|c|c|c|c|c|c|c|c|c|c|c|c|c|}
\hline \multirow[b]{3}{*}{ Antibióticos } & \multicolumn{7}{|c|}{ Idade } & \multicolumn{7}{|c|}{ Gênero } \\
\hline & \multicolumn{3}{|c|}{$\leq 65$ anos } & \multicolumn{3}{|c|}{$>65$ anos } & \multirow[b]{2}{*}{$\mathrm{p}$ valor } & \multicolumn{3}{|c|}{ masculino } & \multicolumn{3}{|c|}{ feminino } & \multirow[b]{2}{*}{$\mathrm{p}$ valor } \\
\hline & $\mathrm{n}^{\circ}$ & $\mathrm{N}$ & $\%$ & $\mathrm{n}^{\circ}$ & $\mathrm{N}$ & $\%$ & & $\mathrm{n}^{\circ}$ & $\mathrm{N}$ & $\%$ & $\mathrm{n}^{\circ}$ & $\mathrm{N}$ & $\%$ & \\
\hline Gentamicina & 66 & 538 & 12,3 & 25 & 217 & 11,5 & 0,572 & 36 & 124 & 20,9 & 65 & 631 & 10,0 & 0,000 \\
\hline Cefalotina & 248 & 533 & 46,5 & 103 & 217 & 47,5 & 0,400 & 75 & 126 & 59,5 & 276 & 624 & 44,2 & 0,007 \\
\hline Cefoxitina & 75 & 510 & 14,7 & 37 & 210 & 17,6 & 0,209 & 35 & 123 & 28,5 & 74 & 597 & 12,4 & 0,000 \\
\hline Nitrofurantoína & 103 & 538 & 19,1 & 65 & 214 & 30,4 & 0,004 & 43 & 123 & 34,9 & 125 & 629 & 19,9 & 0,000 \\
\hline Ácido nalidíxico & 135 & 541 & 24,9 & 76 & 219 & 34,7 & 0,015 & 63 & 125 & 50,4 & 148 & 635 & 23,3 & 0,000 \\
\hline Ceftriaxona & 14 & 538 & 2,6 & 10 & 215 & 4,7 & 0,349 & 14 & 125 & 11,2 & 10 & 628 & 1,6 & 0,000 \\
\hline Ciprofloxacina & 62 & 542 & 11,4 & 39 & 219 & 17,8 & 0,023 & 38 & 125 & 30,4 & 63 & 636 & 9,9 & 0,000 \\
\hline SMZ-TMP & 243 & 522 & 46,6 & 101 & 210 & 48,1 & 0,696 & 58 & 117 & 49,6 & 286 & 615 & 46,5 & 0,758 \\
\hline
\end{tabular}

SMZ-TMP 


\section{DISCUSSÃo}

O presente estudo mostra que Escherichia coli, Proteus e Klebsiella são as três principais bactérias causadoras de ITU adquirida na comunidade na população estudada. Estes resultados estão de acordo com os estudos realizados nas outras regiões do Brasil e do mundo ${ }^{3514}{ }^{16}$. Portanto, os antibióticos usados empiricamente devem ter espectro de ação para enterobactérias, já que são os de maior probabilidade de estarem presentes na ITU adquirida na comunidade.

Os uropatógenos isolados em pacientes deste estudo apresentaram altas taxas de resistência a sulfametoxazoltrimetoprim (46,9\%), cefalotina (46,7\%), ácido nalidíxico $(27,6 \%)$ e nitrofurantoína (22,3\%). Chama a atenção de que esses antibióticos são justamente aqueles mais indicados para o tratamento empírico de ITU adquirida na comunidade em adultos. Os antibióticos menos usados no tratamento ambulatorial de ITU, tais como cefalosporinas de segunda e terceira geração (cefoxitina, ceftriaxona) e aminoglicosídeos (gentamicina), apresentaram taxas inferiores de resistência bacteriana. Esses dados indicam que o emprego freqüente dos antibióticos foi provavelmente o fator responsável pelo surgimento de resistência antimicrobiana. As taxas elevadas de resistência antimicrobiana dos uropatógenos a sulfametoxazol-trimetoprim, cefalotina, ácido nalidíxico e nitrofurantoína também foram observadas nas outras regiões do Brasil ${ }^{216}$. Essas taxas são maiores do que relatadas na América do Norte e Europa ${ }^{168}$. Estes resultados são alarmantes, indicando a necessidade de uso mais racional dos antibióticos no Brasil.

Ácido nalidíxico foi o único antibiótico que apresentou aumento significativo na taxa de resistência bacteriana, com crescimento anual de 5,9\%, durante o período entre 2000 e 2004 . Os antibióticos sulfametoxazol-trimetoprim, cefalotina e nitrofurantoína não apresentaram aumento significativo, mas as prevalências de resistência bacteriana destes antibióticos mantiveram altas durante todo o período estudado. Além disso, ciprofloxacina, um antibiótico recentemente introduzido no tratamento ambulatorial da ITU, mostrou também tendência de aumento na taxa de resistência bacteriana, com um crescimento anual de $3,3 \%$, chegando atualmente a 13,1\%. 0 aumento da resistência bacteriana a ciprofloxacina também foi observado em outra região do Brasil". Não foram encontrados estudos atuais no Brasil sobre evolução temporal da resistência antimicrobiana dos uropatógenos para comparar com os resultados deste estudo. Porém, a manutenção de altas taxas ou a tendência de aumento da taxa de resistência bacteriana no período estudado configura a magnitude do problema da resistência antimicrobiana dos uropatógenos na região extremo sul do Estado do Rio Grande do Sul.

Este estudo mostra que os pacientes masculinos com ITU apresentaram maiores taxas de resistência bacteriana do que as mulheres. Observa-se ainda que os pacientes masculinos tiveram maior prevalência de Proteus e Klebsiella como uropatógenos comparados com as mulheres, e essas bactérias apresentaram maiores taxas de resistência antimicrobiana do que a Escherichia coli. Essa diferença na distribuição etiológica da ITU pode ser um dos fatores responsáveis pela maior taxa de resistência bacteriana em sexo masculino. Os pacientes idosos também apresentaram maior taxa de resistência bacteriana a alguns antibióticos. Porém, não houve diferença significativa quanto à distribuição etiológica entre pacientes com idade maior que 65 anos e aqueles com idade igual ou menor que 65 anos. Estudos futuros são necessários para o melhor entendimento da influência de idade e gênero do paciente na taxa de resistência antimicrobiana dos uropatógenos.

A correlação entre resistência bacteriana in vitro e falha terapêutica em pacientes com ITU ainda não foi adequadamente estudada, porém dois estudos já mostraram pior prognóstico em pacientes que apresentaram resistência bacteriana in vitro a sulfametoxazol-trimetoprim ou trimetoprim e foram tratados empiricamente com esses antibióticos ${ }^{1015} .0$ pior prognóstico foi manifestado por menor taxa de cura clínica e laboratorial, maior duração dos sintomas, maior taxa de retorno para consulta médica e maior frequiência de uso de antibióticos subsequientes. Através do modelo matemático, foram estimadas recentemente, as taxas de cura clínica e laboratorial de ITU tratada com sulfametoxazoltrimetoprim em $88 \%$ e $84 \%$ respectivamente, quando a prevalência de resistência bacteriana in vitro a este antibiótico for de $20 \%$. Até o presente momento, não há critérios universalmente aceitos para estabelecer o limite superior da taxa de resistência bacteriana para indicar os antibióticos no tratamento empírico da ITU. Em 1999, a Sociedade Americana de Doenças Infecciosas sugeriu que sulfametoxazol-trimetoprim deve ser indicado para o tratamento empírico de ITU somente nas regiões onde a prevalência de resistência bacteriana a este antibiótico for inferior a $20 \%{ }^{17}$. Baseado nesse critério, ciprofloxacina é o único antibiótico por via oral que possui o perfil terapêutico adequado para o tratamento empírico dos pacientes adultos com ITU em nossa região. Por outro lado, o uso excessivo de ciprofloxacina irá inevitavelmente acelerar 0 crescimento na taxa de resistência bacteriana a este antibiótico e aumentar também significativamente 0 custo de tratamento de ITU. Esta situação representa um grande desafio aos profissionais médicos para indicar os antibióticos no tratamento empírico de ITU. Considerando-se o custo, disponibilidade e efetividade do tratamento, sugerimos que nos locais com altas taxas de resistência antimicrobiana dos uropatógenos, como nossa região, sulfametoxazol-trimetoprim, cefalosporina de primeira geração, ácido nalidíxico e nitrofurantoína ainda possam ser indicadas no tratamento empírico dos pacientes adultos com ITU adquirida na comunidade, porém, a maior atenção deve ser empregada no acompanhamento desses pacientes. Um retorno em 72 horas após o tratamento deve ser recomendado para avaliar sua resposta terapêutica.

Algumas limitações metodológicas deste estudo devem ser observadas. Os dados foram obtidos em pacientes atendidos num hospital terciário, sendo como um centro médico de referência na região. Portanto, amostra deste estudo não necessariamente representa pacientes com ITU na comunidade. Este estudo foi de natureza retrospectiva baseado no banco de dados eletrônico do hospital. Desta forma, o modo de realização da coleta, bem como as condições de assepsia, a técnica e o local de coleta são 
desconhecidos. 0 mesmo ocorre com o tempo entre a coleta e sua análise no laboratório, não sendo possível afirmar as condições de conservação de todas as amostras de urina neste período. As informações clínicas dos pacientes também não foram contidas no banco de dados eletrônico, e isso impede a investigação dos fatores associados com resistência bacteriana dos uropatógenos, tais como uso prévio de antibióticos, nível socioeconômico, condições de higiene individual, recorrência de ITU e outras doenças concomitantes. Estudos prospectivos e longitudinais devem ser realizados para monitorar a taxa de resistência antimicrobiana dos uropatógenos e para orientar o tratamento empírico dos pacientes com infecção do trato urinário.

\section{AGRADECIMENTOS}

Ao Prof. Dr. Pedro Eduardo Almeida da Silva, Departamento de Patologia da Fundação Universidade Federal do Rio Grande, pela revisão crítica do texto e ao Dr. Róger Giusti Miller, Laboratório de Análises Clínicas do Hospital Universitário da Fundação Universidade Federal do Rio Grande, pelo fornecimento das informações técnicas dos exames microbiológicos.

\section{REFERÊNCIAS}

1. Alós JI, Serrano MG, Gómez-Garcés JL, Perianes J. Antibiotic resistance of Escherichia coli from community-acquired urinary tract infections in relation to demographic and clinical data. Clinical Microbiology and Infection 11: 199-203, 2005.

2. Andrade SS, Sader HS, Jones RN, Pereira AS, Pignatari AC, Gales AC. Increased resistance to first-line agents among bacterial pathogens isolated from urinary tract infections in Latin America: time for local guidelines? Memórias do Instituto Oswaldo Cruz 101: 741-748, 2006

3. Camargo CBS, Pedro CC, Lourenço DS, Gironi RHAR, Martinez R. Infecçäo de vias urinárias na comunidade de Ribeiräo Preto, SP: etiologia, sensibilidade bacteriana a antimicrobianos e implicaçöes terapêuticas. Medicina (Ribeirão Preto) 35: 173-178, 2002.

4. Erb A, Stürmer T, Marre R, Brenner H. Prevalence of antibiotic resistance in Escherichia coli: overview of geographical, temporal, and methodological variations. European Journal of Clinical Microbiology \& Infectious Diseases 26: 83-90, 2007.

5. Gales AC, Sader HS, Jones RN, SENTRY Participants Group (Latin America) Urinary tract infection trends in Latin American hospitals: report from the SENTRY antimicrobial surveillance program (1997-2000). Diagnostic Microbiology and Infectious Diseases 44: 289-299, 2002.

6. Grude N, Tveten Y, Jenkins A, Kristiansen BE. Uncomplicated urinary tract infections. Bacterial findings and efficacy of empirical antibacterial treatment. Scandinavian Journal of Primary Health Care 23: 115-119; 2005.

7. Herlberg IP, Schor N. Aborgagem Diagnóstica e Terapêutica na Infecção do Trato Urinário -ITU. Revista Associação Médica Brasileira 49: 109-116, 2003.

8. Karlowsky JA, Kelly LJ, Thornsberry C, Jones ME, Sahm DF. Trends in antimicrobial resistance among urinary tract infection isolates of Escherichia coli from female outpatients in the United States. Journal of Urology 170: 335-336, 2003.

9. Lopes AA, Salgado K, Martinelli R, Rocha H. Aumento da frequiência de resistência à norfloxacina e ciprofloxacina em bactérias isoladas em uroculturas. Revista da Sociedade Médica Brasileira 44: 196-200 1998.

10. McNulty CA, Richards J, Livermore DM, Little P, Charlett A, Freeman E, Harvey I, Thomas M. Clinical relevance of laboratory-reported antibiotic resistance in acute uncomplicated urinary tract infection in primary care. Journal of Antimicrobial Chemotherapy 58: 1000-1008, 2006.

11. National Committee for Clinical Laboratory Standards. Performance standards for antimicrobial disk susceptibility tests. NCCLS Document M2-A7. Wayne, PA, USA, 2000.

12. National Committee for Clinical Laboratory Standards. Supplemental tables: disk diffusion. NCCLS Document M100-S10. Wayne, PA, USA, 2000.

13. National Committee for Clinical Laboratory Standards. Methods for dilution antimicrobial susceptibility tests for bacteria that grow aerobically. NCCLS Documents M2-A8 and M100-S13. Wayne, PA, USA, 2003.

14. Queiroz K, Poletto RC. Suscetibilidade antimicrobiana de uropatógenos em pacientes ambulatoriais na Cidade de Goiânia, GO. Revista da Sociedade Brasileira de Medicina Tropical 38: 416-420, 2005.

15. Raz R, Chazan B, Kennes Y, Colodner R, Rottensterich E, Dan M, Lavi I, Stamm W, Israeli Urinary Tract Infection Group. Empiric use of trimethoprimsulfamethoxazole (TMP-SMX) in the treatment of women with uncomplicated urinary tract infections, in a geographical area with a high prevalence of TMP-SMX resistant uropathogens. Clinical Infectious Diseases 34: 1165-1169, 2002.

16. Sociedade Brasileira de Urologia. Diretrizes em urologia: Infecção do Trato Urinário. Disponível no site da Sociedade Brasileira de Urologia: http://www. sbu.org.br/socios/diretrizes/inf_urinaria.pdf, 2004.

17. Warren JW, Abrutyn E, Hebel JR, Johnson JR, Schaeffer AJ, Stamm WE. Guidelines for antimicrobial treatment of uncomplicated acute bacterial cystitis and acute pyelonephritis in woman. Infectious Diseases Society of America (IDSA). Clinical Infectious Diseases 29: 745-758, 1999. 